\title{
Effect of Arginine on IL-6, IL-17 and TGF- $\beta$ levels in high-fat Diet-Induced Hypercholesterolemia Rat
}

\author{
Eryati Darwin', Eka Fithra Elfi², Dachriyanus*3 \\ 'Department of Histology and Immunology, Faculty of Medicine Andalas University, Padang, INDONESIA. \\ 2Department of Cardiology, Faculty of Medicine Andalas University, Padang, INDONESIA. \\ ${ }^{3}$ Faculty of Pharmacy Andalas University, Padang, INDONESIA.
}

\begin{abstract}
Objective: The aim with the present study was to determine that arginine affect to the level of IL-6, IL-17 and TGF- $\beta$ in high-fat diet-induced hypercholesterolemia in rats. Material and Methods: Male Wistar rats in eight weeks old $(n=35)$ were divided into five groups, one group as a control (C) were given standard $r$ fed and four groups were given high-fat diet $(\mathrm{Hfd})$ with fats from beef tallow. One group of high-fat diet rats recieved no arginine, three groups were recieved arginine in doses of 100, 200 and $400 \mathrm{mg} / \mathrm{kg} / \mathrm{day}$ each for three weeks. After two weeks of treatment, blood cholesterol were measured, while serum level of IL-6, IL-17 and TGF- $\beta$ were measured the final five weeks of this protocol by ELISA methods. Results: There were no significantly different between groups, which received different doses of arginine $(p>0,05)$. IL-17 of high-fat diet received arginine of doses 100 and $200 \mathrm{mg} / \mathrm{kg} /$ day were significantly higher compared to high-fat diet received no arginine and control group. Interestingly,
\end{abstract}

IL-17 in high-fat diet group received arginine of doses $400 \mathrm{mg} / \mathrm{kg} /$ day were lower compared to groups received lower doses of arginine. TGF- $\beta$ levels in the high-fat diet group's recieved arginine were lower compared to control group. Conclusions: The effect of arginine on cytokines plays the role on vascular inflammation due to high-fat diet.

Key words: Arginine, high-fat diet, IL-6, IL-17, and TGF- $\beta$

Correspondence :

Dachriyanus,

Faculty of Pharmacy Andalas University, Padang, INDONESIA.

E-mail: dachriyanus@ffarmasi.unand.ac.id

DOI: 10.5530/jyp.2017.9.16

\section{INTRODUCTION}

Food contains nutritional components that can be categorized by macronutrients including carbohydrate, fat and protein along with micronutrients. Dietary fat is a needed for energy, cellular membranes, biosynthesis of hormone and media for fat-soluble vitamins. Postprandial lipidemia is a physiological response after consuming a meal-contained fat, include increasing concentration of triglyceride (TG), very low-density lipoprotein (VLDL) cholesterol, and chylomicron; decreasing concentration of highdensity lipoprotein (HDL) cholesterol; and has no effect on concentration of low-density lipoprotein (LDL) cholesterol. ${ }^{1}$

The high-fat diet leads to distributional shifts of VLDL, LDL and HDL particle sizes, numbers, and plasma concentrations. The various particle sizes demonstrated both pro-atherogenic (large VLDL and small LDL) and anti-atherogenic (large LDL) effects. ${ }^{2}$ A high-fat diet is usually followed by transient endothelial dysfunction in association with raised triglyceride-rich lipoproteins, lead to obesity that induce vasculature oxidative stress which contributes to endothelial dysfunction and high blood pressure as a major risk factor for the development of cardiovascular diseases. ${ }^{3}$ Abnormal vasoactivity after a fatty meal is reduced by addition of antioxidants to the meal, showing that postprandial oxidative stress plays an important role. ${ }^{4}$ However the effects of high-fat diet on vascular oxidative status and endothelial function remain unknown. ${ }^{5}$

The healthy vascular endothelium senses mechanical stimuli, such as pressure and shear stress, and hormonal stimuli such as vasoactive substances by releases agents that regulate vasomotor function, trigger vascular wall inflammatory processes, and affect hemostasis. A wide range factors are produced to regulate vascular tone, structure and exerts anticoagulant, antiplatelet, fibrinolytic properties, cellular adhesion, smooth muscle cell proliferation, and vessel wall inflammation. ${ }^{6}$ The maintenance of vascular tone is achieved with the release of various dilator and constrictor substances. Nitric oxide (NO) is the main vasodilative substance released by the endothelium. the other endothelium-derived vasodilators are prostacyclin and bradykinin. Prolonged and or repeated exposure for cardiovascular risk factors may reduce the protective effect of endogenous system of anti-inflammatory in endothelial cells. As a result, endothelial cell become dysfunctional and also lose integrity, progress to aging , as well as drop it onto the circulation. ${ }^{6}$

$\mathrm{NO}$ is produced in the endothelium by conversion L-arginine into L-citrulline by the enzyme constitutive, endothelial nitric oxide synthase (eNOS). Although both the L- and D-enantiomers of arginine are present in the human circulation, only L-arginine is used by eNOS as the substrate for production of NO. NO plays an important role to maintain the homeostasis of the cardiovascular system to regulate vasodilation.? Moreover NO plays a role for angiogenesis, inhibit the proliferation of smooth muscle cells of blood vessels, reduce the expression of adhesion molecules and inhibit the release of endothelin-1, which plays a role in the mechanisms of vasoconstriction of blood vessels. ${ }^{8}$ L-arginine is considered as the substrate for eNOS and very important for endothelial health. A number of initial experiments using animal models to investigate the efficacy of potential supplementation of L-arginine in reversing endothelial dysfunction in pathological states. Numerous studies that support the benefits of supplementation with L-arginine acute and chronic endothelial NO production were carried out in animal models and human subjects. ${ }^{9}$ Clinical trials using L-arginine in the treatment of coronary artery and peripheral artery disease, as well as in the prevention of in-stent restenosis were carried out. The mechanism of benefit of L-arginine on endothelial function is not clear, because the concentration of intracellular L-arginine far exceeded that required by eNOS. $\mathrm{L}$-arginine in atherosclerosis patients could elevated levels of asymmetric 
dimethylarginine, an endogenous inhibitor of eNOS. L-arginine supplementation may be needed in special conditions such as senile dementia, erectile dysfunction, and male infertility, malnutrition, ammonia production, burns, infections, peritoneal dialysis, rapid growth, sepsis and improving athletic performance. ${ }^{10}$

Endothelial dysfunction has been proposed to be an early symptom of pathophysiological importance in the atherosclerotic process. Low-density lipoprotein (LDL) modified by oxygen radicals to oxidized LDL (oxLDL), which in turn induces endothelial cells to express adhesion molecules. Monocytes and $\mathrm{T}$ cells bind to adhesion molecules-expressing endothelial cells and respond to locally produced chemokine's by migrating into the sub endothelial tissue. Scavenger receptor mediated macrophage uptake of ox - LDL particles, which causes the accumulation of intracellular cholesterol and the formation of foam cells and promote the production of many pro-inflammatory cytokines including interferon- $\alpha$ (IFN- $\alpha$ ) and tumor-necrosis factor (TNF). ${ }^{11}$ These cytokines and others prompt macrophage activation, production of other pro-inflammatory mediators, activate endothelial cells, increased expression of adhesion - molecule and the propensity of thrombus formation and activate proliferation of smooth muscle - cells and collagen production. ${ }^{12}$ Cytokines and growth factors cause the proliferation of smooth muscle that will lead to atherosclerotic plaque. Plaque inflammation might be attenuated in response to the anti-inflammatory cytokines Interleukin-10 (IL-10) and transforming growth factor- $\alpha$ (TGF- $\alpha$ ), which are produced by several cell types including regulatory $\mathrm{T}$ (Treg) and helper $\mathrm{T}(\mathrm{Th})$ cells, macrophages, and for TGF- $\alpha$, also vascular cells and platelets. Morever, macrophages also produce anti-inflammatory cytokines IL-10 and TGF- $\beta$ which triggers Treg differentiation. ${ }^{13}$

Vascular endothelial response to high level of blood cholesterol was marked with the level of cytokines IL-6, IL- 17 and TGF- $\beta$. Interleukin- 6 and TGF- $\beta$ triggers Th17 to produce IL-17 and IL-10, which in this case IL-17 can induce anti-inflammatory effects through suppression of Th1 cell differentiation pathogens. ${ }^{14}$ Interleukin-17 is a pro-inflammatory cytokine that has six isoforms with five receptor subunits. Cells can be activated by IL-17 is the epithelial cells, endothelial, and fibroblasts, as it also macrophages and dendritic cells. ${ }^{15}$ Interleukin-17 pathway activation that occurs through nuclear factor (NF)- $\kappa B$ causes the gene expression of pro-inflammatory cytokines such as tumor necrosis factor- $\alpha$ (TNF- $\alpha$ ), IL-1, IL-6, and granulocyte-macrophage colony-stimulating factor (GM$\mathrm{CSF}$ )], chemokine, matrix metalloproteinase and antimicrobial peptides. Interleukin-17 increases granulocyte colony-stimulating factor (G-CSF) for the recruitment of neutrophils to sites of inflammation. This is related to the notion that IL-17 plays a role in the formation of atherosclerotic plaque. ${ }^{16}$ Interleukin- 6 is a pleiotropic cytokine, secreted by cells of the immune system such as non-specific dendritic cells, monocytes, macrophages mast cells, B lymphocytes, fibroblasts, endothelial, keratinocytes and activated T cells. Many studies have shown that IL-6 plays an important role to regulate the balance between Th17 and Treg which produces IL-17. ${ }^{17}$ Furthermore, IL-6 and IL-23 in combination with IL- $1 \beta$ and Transforming growth factor $\beta$ (TGF- $\beta$ ) were able to induces the differentiation of naive $\mathrm{T}$ become Th17. By contrast, IL- 6 inhibits the differentiation of naive $\mathrm{T}$ into Treg. Transforming growth factor $\beta$ is a multifunctional cytokine, produced by non-immune cells and immune, including macrophages. While it is known that TGF- $\beta$ plays an important role in the regulation of cellular, but much is still unknown pathway activation. Proteases, integrin's, $\mathrm{pH}$ and reactive oxygen species (ROS) is a factor known to play a role in activating TGF- $\beta .^{18}$

In this study, the effect of arginine on IL-6, IL-17 and TGF- $\beta$ levels in high-fat diet-induced hypercholesterolemia in rat was reported.

\section{MATERIAL AND METHODS}

This study was carried out on eight weeks old male Rattusnorvegicus strain Wistar with a body weight of 250-300 grams. The rats were obtained from animal breeding in Faculty of Pharmacy Andalas University, acclimatized for one week in a room temperature with ad libitum access to water and food. There were 35 of rats that divided into five groups, one group of rats as a control group (C) were given standard rats fed, whereas four groups of rats were given high-fat diet-fed with fats from beef tallow. One group of high-fat diet rats revcieved no L-arginine (Hfd), while three groups were recieved L-arginine (Sigma Aldrich, St. Louis, MO, USA) in different doses of $100 \mathrm{mg} / \mathrm{kg}$ body weight/day, $200 \mathrm{mg} / \mathrm{kg}$ body weight/day and $400 \mathrm{mg} / \mathrm{kg}$ body weight/day (Hfd+Arg100, Hfd+Arg200 and $\mathrm{Hfd}+\mathrm{Arg} 400)$. L-arginine was disolved in water and given orally for three weeks. After two weeks of this protocol, $1 \mathrm{ml}$ blood were collected from medial canthus sinus orbaitalis to determine the level of total cholesterol (Microlab 300). At the final five weeks of this protocol, rats were cervical dislocated under ketamine annesthesia. Blood was colected from the heart to determine serum level of IL-6, IL-17 and TGF- $\beta$ (Bio Legend) by ELISA methods.

The procedures of this study were approved by the Ethical Committee of Faculty of Medicine Andalas University (Reg.067/2015)

\section{Data Analysis}

Data were presented as mean \pm standard deviation (SD). Statistical analysis was performed using one-way analysis of variants (ANOVA). When data were in normal distribution, then followed by multiple comparisons Post Hock Test Benferonni. A value of $\mathrm{P}<0,05$ indicates statistical significance.

\section{RESULTS}

Our results shows that at the end of five weeks study, the body weight of the rats were increase on the average 6-8 \% compared to baseline body weight. Blood total cholesterolthat were measured after two weeks of this protocol to difine hypercholesterolemia was shown in table 1. Total cholesterol level of the groups of rats with a high-fat diet (Hfd, $\mathrm{Hfd}+\operatorname{Arg} 100, \mathrm{Hfd}+\operatorname{Arg} 200$ and $\mathrm{Hfd}+\operatorname{Arg} 400)$ were significantly higher compared to the control group received standard fed $(\mathrm{C})(\mathrm{p}<0,05)$, but no significantly different between groups $(\mathrm{p}>0,05)$

Serum level of IL-6, IL-17 and TGF- $\beta$ shown in table 2 . Using the Kolmogorov Smirnoftest was known that levels have IL-6 IL-17 and TGF- $\beta$ was in a normal distribution, so that the data was processed using one way ANOVA test. In high-fat diet groups of rats received no arginine (Hfd), the IL-6 level was significantly higher compared to control group, and it wasalso significantly higher compared to the groups received different doses of arginine $(\mathrm{p}<0,05)$. The level of IL- 6 in high-fat diet group of rats received arginine were significantly lower compared to group received no arginine (Hfd) and control group, and there were no

\begin{tabular}{|c|c|c|}
\hline No & Groups & Mean \pm SD \\
\hline 1 & C & $55.60 \pm 2.61$ \\
\hline 2 & Hfd & $88.60 \pm 8.03$ \\
\hline 3 & $\mathrm{~T} 2$ & $86.70 \pm 6.63$ \\
\hline 4 & T3 & $83.44 \pm 8.40$ \\
\hline 5 & T4 & $90.43 \pm 6.05$ \\
\hline
\end{tabular}


Dachriyanus et al: Effect of arginine on hypercholesterolemia rat

Table 2 : Serum levels of IL-6, IL-17 and TGF- $\beta$ in the control Group (C), high-fat diet group recieved no arginine (Hrd), and high-fat diet group recieved arginine of doses $100 \mathrm{mg} / \mathrm{kg} /$ day (Hrd+Arg100), $200 \mathrm{mg} / \mathrm{kg} /$ day (Hrd+Arg200) and 400 mg/kg/day (Hrd+Arg400)

\begin{tabular}{ccccc}
\hline No & Group & IL-6 $(\rho g / m l)$ & IL-17 $(\rho g / m l)$ & TGF- $\beta(\rho g / m l)$ \\
\hline 1 & C & $35.20 \pm 13.59$ & $0.95 \pm 0.56$ & $18.06 \pm 10.97$ \\
2 & Hrd & $98.72 \pm 13.59$ & $0.96 \pm 0.88$ & $11.49 \pm 4.92$ \\
3 & Hrd+Arg100 & $17.72 \pm 17.95$ & $9.64 \pm 2.86$ & $13.27 \pm 5.96$ \\
4 & Hrd+Arg200 & $15.49 \pm 5.09$ & $7.21 \pm 4.09$ & $13.39 \pm 4.52$ \\
5 & Hrd+Arg400 & $27.60 \pm 18.88$ & $0.68 \pm 0.22$ & $17.59 \pm 7.37$ \\
\hline
\end{tabular}

Average values in the same column followed by different superscript are significantly different $(\mathrm{p}<0,05)$

significantly different between group received arginine in different doses (Hfd+Arg100, Hfd+Arg200 and Hfd+Arg400).

The level of L-17 in high-fat diet groups of rats received arginine of doses 100 and $200 \mathrm{mg} / \mathrm{kg} /$ day (Hfd+Arg100 and Hfd+Arg200) were significantly higher compared to high-fat diet group received no arginine, high-fat diet group received arginine of doses $400 \mathrm{mg} / \mathrm{kg} /$ day and control group. The levels of TGF- $\beta$ in high-fat diet groups of ratstend to be lower compared to control group, but there were no significantly different ( $p>0,05)$. There were no significantly different between groups in high-fat diet groups received arginine.

\section{DISCUSSION}

Prolonged feeding with fat-enriched diets induces an increase in body weight in susceptible rats in the range of $10 \%$ to $20 \%$ over standard chow-fed controls. In this study, an increase in body weight in a high-fat dietgroups of rats was in the range $6 \%-8 \%$. That could be because the period of high-fat fed was five weeks, begins at the age of eight weeks. In high-fat diet rats the increasing of body weight probably as a consequence of changes in lipid metabolism and storage. Other study showed that high-fat diet rats for 30 weeks developed obesity characterized by increase in final body weight and fat-pad mass. Obesity induction is most effective when the diet is started at a young age and continues for several weeks. ${ }^{19}$

From these results, giving a high-fat diet fed derived from beef tallow for two weeks has increase blood cholesterol levels of the rats in. Beef tallow contains saturated fatty acids and cholesterol, whereas observational studies have shown that high intake of saturated fatty acids is positively associated with increasing levels of blood cholesterol and high coronary heart disease mortality rate. ${ }^{20}$ Saturated fatty acids will be absorbed in the gastrointestinal tract in the form of chylomicrons and enter the bloodstream. On the surface of endothelial lipoprotein lipase enzyme catalyzes the breakdown of triglycerides within chylomicrons to fat-free and energy. High fat consumption significantly not only increases LDL cholesterol levels, but at the same time increasing blood HDL cholesterol levels and thus automatically increasing total blood cholesterol levels. Several study shows showed that administration of fat in experimental animals for 18 days increases total blood cholesterol levels and triglycerides. ${ }^{19}$ While the other study by providing a high-fat diet and high cholesterol for three weeks in baboons, found that there was an increase in total cholesterol, LDL and oxidized LDL. ${ }^{2}$

A number of experimental studies in rats have reported that high-fat diet induces obesity and promotes metabolic and vascular alteration. Vascular endothelial response to high level of blood cholesterol in our study was marked with the level of cytokines IL-6, IL-17 and TGF- $\beta$. Interleukin- 6 TGF- $\beta$ and IL-6 triggers Th17 to produce IL- 17 and IL-10, which in this case IL-17 can induce anti-inflammatory effects through suppression of Th1 cell differentiation pathogens. ${ }^{16}$ The level of IL-6 in a high-fat diet were higher compared to the control group received standard diet fed. LDL cholesterol activates endothelial cells, macrophages, lymphocytes, fibroblast and vascular smooth muscle to secrete Il-6. IL-6, as soluble mediator of the inflammatory responsewill diffuse from sub mucous lining into the vascular lumen, ${ }^{21}$ so that the high levels of IL-6 was detected in the blood of high-fat diet rats.Arginine is a nitric oxide precursor, has an indirect antioxidant role and is a naturally occurring continuously released vasoactive agent that controls vascular tone. In our study, the level of IL- 6 in the high-fat diet rats supplemented with arginine was lower compared to the high-fat diet rats with no arginine. Arginine may reduces oxidative stress and scavenges superoxide radicals and increase NO that reduced due to the inflammatory process, which augments endothelium-dependent vasodilatation in hypercholesterolemia rats. Study in patients with acute myocardial found that l-arginine plays a significant role in reducing the modified serum cholesterol levels and promotes the elevation of HDL cholesterol arginine reduces oxidative stress and scavenges superoxide radicals. ${ }^{10}$ Arginine has a vasomotor effect through the production of endothelial derived relaxing factor i.e. NO. Long term oral arginine administration in patients with hypercholesterolemia, hypertension, and smoking may improve endothelial function, but had no effect on endothelial function of patients with diabetes mellitus. ${ }^{22}$

Cholesterol is anatherogenic factor to the blood vessels, which triggered non-specific immune response nor specific.In this study the levels of IL-17 in high-fat diet rats received low doses of arginine were higher than standard-diet fed rats. Interestingly, IL-17 level in high-fat diet group of rats received highest doses of arginine $(400 \mathrm{mg} / \mathrm{kg} /$ day) were lower compared to the high-fat diet rats received lower doses of arginine. It was due to The presence of high level od IL- 6 and TGF $\beta$ suppresses Th-17 development that decreased IL-17 production. IL- 6 and TGF $\beta$ are critical for Th17 differentiation in vitro and in vivo. ${ }^{12}$ IL-17 is predominantly a pro-inflammatory cytokine, it has pleiotropic and environment-specific functions. But the function of IL-17 remains controversial, while one study found that IL-17 is protective against atherosclerosis, several other recent studies have suggested that IL-17 plays a pro-atherogenic role. ${ }^{13}$

Induction of IL-17 in the presence of anti-inflammatory cytokines will provide a protective effect and regulation. In this study, a doses an periods of arginine supplementation play a role to possess numerous useful physiological properties and immunomodulatory effects possibly by increasing NO secretion. A study demonstrate that iNOS is expressed in activated $\mathrm{CD}_{4}^{+} \mathrm{T}$ cells, and $\mathrm{NO}$ derived from iNOS in activated $\mathrm{CD} 4^{+}$ $\mathrm{T}$ cells suppresses Th17 cell development. Their results support the concept that iNOS expressed by $\mathrm{T}$ cells may play an important role in the development of inflammatory diseases by controlling Th17 immune responses. ${ }^{23}$ Recently published that loss of suppressor of cytokine signaling-3 (SOCS-3) in mouse T cells increases both IL-17A and IL-10 production, inducing an anti-inflammatory macrophage phenotype, which results in a reduction in lesion development and vascular inflammation. This suggests that IL-17 may have a protective role in atherogenesis, while other study found that IL-17 also enhanced the in vivo immunosuppressive effect of mesenchymal stem cells in an iNOS-dependent 
manner. ${ }^{14}$ The atheroprotective effect of IL-17 seems to be mediated at least in part by the regulation of other cytokines and by its inhibitory effect on VCAM-1 expression, an adhesion molecule mediating the accumulation of monocytes and $\mathrm{T}$ cells within the lesions. ${ }^{9}$

TGF- $\beta$ is a pleiotropic cytokine with potent regulatory and inflammatory activity. The multi-faceted effects of TGF- $\beta$ on numerous immune functions are cellular and environmental context dependent. These results shows that the levels of TGF- $\beta$ in the high-fat diet rats were lower in compared to the control group. LDL cholesterol as an autoantigen induces chronic inflammatory response whereas TGF- $\beta$ plays a major role as regulator. TGF- $\beta$ in the presence of IL- 6 drives the differentiation of $\mathrm{T}$ helper 17 (Th17) cells, which can promote further inflammation and augment autoimmune conditions. Severe hypercholesterolemia can induce a switch of autoimmune responses from Th1 to Th2 effector type in atherosclerotic knockout mice and led to elevated circulating TGF $\beta-1$ level. ${ }^{24}$ TGF- $\beta$ may increase the stability of plaques by inhibiting $\mathrm{T}$ cell responses and macrophage activation and by stimulating collagen synthesis. Through NO, arginine supplementation in this study may play a role in reducing ROS formation reduces endothelial inflammation, actively reduces LDL cholesterol and plays a vital role in attenuating the damage being caused due to oxidation of lipids. ${ }^{15}$ High doses of arginine play a role to induce inflammation due to the high level of IL- 6 and low level of IL-17. TGF- $\beta$ seem also induced to suppress the inflammatory process.

\section{CONCLUSIONS}

These study demonstrate that the arginine affect to cytokines that play a role in vascular inflammation due to high-fat diet.

\section{ACKNOWLEDGEMENT}

We thank Mrs. Dessy Arisanty, from Faculty of Medicine, Andalas University for her help

\section{FINANCIAL SUPPORT AND SPONSORSHIP}

We thank Faculty of Medicine, Andalas University for Financial Support

\section{CONFLICTS OF INTEREST}

There is no conflict of interest.

\section{ABBREVIATIONS USED}

TG: Triglyceride; VLDL: Very low-density lipoprotein; HDL: Highdensity lipoprotein; LDL: Low-density lipoprotein; oxLDL: Oxidized LDL; NO: Nitric oxide; eNOS : Endothelial nitric oxide synthase; IFN- $\alpha$ : Interferon- $\alpha$; TNF: Tumor-necrosis factor; IL-10: Interleukin-10; TGF- $\alpha$ : Transforming growth factor- $\alpha$; Treg: Regulatory T; Th: T Helper; NF: Nuclear factor; GM-CSF: Granulocyte-macrophage colony-stimulating factor; G-CSF: Granulocyte colony-stimulating factor; Hfd: High-fat diet rats received no L-arginine; Arg: Arginine; ANOVA: Analysis of variance.

\section{REFERENCES}

1. Ebbert JO and Jensen MD. Fat Depot, free fatty acids, and dyslipidemia. Nutrients. 2013;5:498-509.

2. Wojczynski MK, Glasser SP Oberman A, Kabagambe EK, Hopkins PN, Tsai MY et al. High-fat meal effect on LDL, HDL, and VLDL particle size and number in the Genetics of Lipid-Lowering drugs and diet network (GOLDN): an interventional study. Lipids in Health Dis. 2011;10:181-92.

3. Osfor MM, Kensarah O, Azzeh F, Elmadbouly MA, El-Qutry M. Biochemical changes in lipid and carbohydrate metabolism in obese diabetic male albino rats. Int J Nutr Food Sci. 2013;2(2):24-30.

4. Sies H, Stahl W, Sevianian A. Nutritional, Dietary and Postprandial Oxidative Stress. J Nutr. 2005:135(5):969-972.

5. Shi Q, Vandeberg JF, Jett C, Rice K, Leland MM, Talley L, et al. Arterial endothelial dysfunction in baboons fed a high-cholesterol, high-fat diet. Am J Clin Nutr. 2005;82(4):751-9.

6. Libby P, Ridker PM, Hansson GK. Progress and challenges in translating the biology of atherosclerosis. Nature. 2011;473:317-25.

7. Böger RH. The Pharmacodynamics of I-Arginine J. Nutr. 2007;137(6):1650-5.

8. Gornik HL, Creager MA. Arginine and Endothelial and Vascular Health. J Nutr. 2004;134(10):2880-7.

9. Alvares TS, Conte-Junior CA, Silva JT, Paschoalin VMF. Acute L-Arginine supplementation does not increase nitric oxide production in healthy subjects. Nutr Metabolism. 2012;9:54-62.

10. Liuzzo G, Trotta F, Pedicino D. Interleukin-17 in atherosclerosis and cardiovascular disease: the good, the bad and the unknown. Eur Heart J. 2013;34:556-9.

11. Deanfield JE, Halcox JP, Rabelink TJ. Endothelial Function and Dysfunction, Testing and Clinical Relevance. Circulation. 2007;115:1285-95.

12. Oufella HI, Taleb S, Mallat Z, Tedgui. Recent Advances on the Role of Cytokines in Atherosclerosis. Arteriosclerosis, Thrombosis, and Vascular Biology. 2011;31:969-79.

13. Zhou X, Johnston TP, Johansson D, Parini P, Funa K, Svensson J et al. Hypercholesterolemia leads to elevated TGF-beta1 activity and T helper 3-dependent autoimmune responses in atherosclerotic mice. Atherosclerosis. 2008;204(2):381-7.

14. Nascimento TBB, Baptista RFF, Pereira PC, Campos DH, Leopoldo AS, Leopoldo $A P L$, et al. Vascular alterations in high-fat diet-obese rats: role of endothelial L-arginine/NO pathway. Arq Bras Cardiol. 2011;97(1):40-5.

15. Gaffen SL. An Overview of IL-17 Function and Signaling. Cytokine. 2008;43(3):402-7.

16. Kleinewietfeld M, Manzel A, Titze J, Kvakan H, Yosef N, Linker RA, et al. Sodium chloride drives autoimmune disease by the induction of pathogenic $\mathrm{TH} 17$ cells. Nature. 2013:496:518-22.

17. Zaragoza YFZ, Martínez EJR, Avelar MD, Magallanes NG, Pérez1 NV,2014. Th17 Cells in Autoimmune and Infectious Diseases. Int J Inflam. 2014:778-90.

18. Gutcher I, Donkor MK, Ma Q, Rudensky AY, Flavell RA, Li MO. Autocrine transforming growth factor- $\beta 1$ promotes in vivo Th17 cell differentiation. Immunity. 2011;34:396-408.

19. Tousoulis D, Antoniades C, Tentolouris C, Goumas G, Stefanadis C, Toutouzas P. L-Arginine in cardiovascular disease: dream or reality?. Vasc Med. 2007;203-11.

20. Legrand P, Rioux V. The Complex and Important Cellular and Metabolic Functions of Saturated Fatty Acids. Lipids. 2010;45(10):941-6.

21. Pineiro V, Ortiz-Moreno A, Mora-Ewscobedo R, Hernandes-Navaro MD. Effect of L-arginine Oral Supplementation on Response to Myocardial Infarction in Hypercholesterolemic and Hypertensive Rats. Plant Foods Hum Nutr. 2010;65(1):31-7

22. Loscalzo J. Adverse Effects of Supplemental I-Arginine in Atherosclerosis Consequences of Methylation Stress in a Complex Catabolism?. Art Throm Vas. 2003;23:3-5.

23. Das J, Ren G, Zhang L, Roberts Al, Zhao X, BothwellAL, et al. Transforming growth factor beta is dispensable for the molecular orchestration of Th17 cel differentiation. J Exp Med. 2009;206:2407-16.

24. Endemann DH, Schiffrin EL. Endothelial Dysfunction. J Am Soc Nephrol. 2004;15(8):1983-92

Article History: Submission Date: 02-07-16; Revision Date: 30-07-16; Accepted Date: 02-09-16.

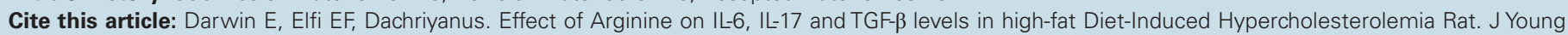
Pharm. 2017;9(1):83-6. 